\title{
Sex Differences in Cause-specific Mortality in Japanese Dialysis Patients
}

\author{
Minako Wakasugi ${ }^{1}$ and Ichiei Narita ${ }^{2}$
}

\begin{abstract}
:
Objective The survival advantage of females over males is lost in dialysis patients in many countries. Japanese female hemodialysis patients, however, have a survival advantage over their male counterparts. This study explored causes of death that contribute to sex differences in all-cause mortality in Japanese dialysis patients.

Methods Data from the Japanese Society for Dialysis Therapy registry and National Vital Statistics from 2017 and 2018 were used. Standardized mortality ratios, male-to-female mortality rate ratios, and ageadjusted differences between sexes were calculated for all-cause, cardiovascular, and non-cardiovascular mortality, as well as cause-specific mortality, in dialysis patients and the general population.

Results During the 2-year study period, 41,006 and 21,254 deaths occurred in 417,740 and 225,292 patient-years in male and female dialysis patients, respectively. The age-standardized all-cause mortality ratio was 1.21 (95\% confidence interval, 1.20-1.23) for male patients compared to female patients. The male-tofemale mortality rate ratio for cardiovascular disease was about 1.4 in younger age categories but closer to 1.0 in older age categories. Conversely, the ratio for non-cardiovascular disease was about 1.3 in older age categories but closer to 1.0 in younger age categories. Death from infectious disease, malignancy, and heart failure contributed to $38.4 \%, 22.7 \%$, and $12.1 \%$, respectively, of the male-to-female difference in all-cause mortality of dialysis patients.

Conclusion Low cardiovascular mortality in younger age categories and low non-cardiovascular mortality in older age categories contributed to the survival advantage of female Japanese dialysis patients. Infectious disease was the greatest contributor to sex differences in all-cause mortality.
\end{abstract}

Key words: cardiovascular, dialysis, epidemiology, gender, infection, non-cardiovascular

(Intern Med Advance Publication)

(DOI: 10.2169/internalmedicine.8981-21)

\section{Introduction}

The survival advantage of females over males, a generally observed phenomenon, essentially disappears in both incident dialysis patients (1) and prevalent hemodialysis patients (2) but is partly preserved in prevalent hemodialysis patients in some European countries and Japan (2). For instance, a European cohort study of incident adult dialysis patients using data from the European Renal AssociationEuropean Dialysis and Transplant Association (ERA-EDTA) Registry found that males and females starting dialysis die at the same rate (1). Furthermore, a study using data from the international Dialysis Outcomes and Practice Patterns Study (DOPPS) from 12 countries (Australia, Belgium, Canada, France, Germany, Italy, New Zealand, Spain, Sweden, the United Kingdom, the United States, and Japan) reported that mortality rates in male and female prevalent hemodialysis patients were very similar, with male-to-female mortality rate ratios (MMRs) very close to 1.0 in the 5 assessed age groups (2). When analyzed by country, however, a slightly higher mortality risk for males was observed in Belgium, France, Germany, Australia/New Zealand, and Japan (2).

The causes of death that contribute to the slightly higher

${ }^{1}$ Department of Inter-Organ Communication Research, Niigata University Graduate School of Medical and Dental Sciences, Japan and ${ }^{2}$ Division of Clinical Nephrology and Rheumatology, Niigata University Graduate School of Medical and Dental Sciences, Japan Received: November 8, 2021; Accepted: January 17, 2022; Advance Publication by J-STAGE: February 26, 2022 Correspondence to Dr. Minako Wakasugi, minakowa@med.niigata-u.ac.jp 
mortality risk for males in these countries are unclear. Studies examining specific causes of death would help identify the relative impact of various diseases on sex differences in mortality. Furthermore, a careful analysis of this aspect can help establish future strategies to lower mortality rates in dialysis patients.

The objectives of this study were to determine the MMRs for all-cause, cardiovascular, and non-cardiovascular mortality using data from a national registry of dialysis patients (annual survey of the Japanese Society for Dialysis Therapy Renal Data Registry [JRDR]) and to determine the extent to which (certain categories of) diseases contribute to sex differences in mortality. We also calculated the all-cause, cardiovascular, and non-cardiovascular mortality rates as well as MMRs of the general Japanese population using National Vital Statistics data.

\section{Materials and Methods}

\section{Data Source}

The mortality data of dialysis patients were extracted from annual data reported by the JRDR for 2017 and $2018(3,4)$ using the Web-based Analysis of Dialysis Data Archives (WADDA) system. Details of JSDT registry data collection techniques and characteristics of this dialysis population have been described elsewhere $(3,4)$. The JRDR collects data on the medical history, treatment conditions, and outcomes of individual dialysis patients from dialysis facilities in Japan every year by questionnaire. According to annual data reported by the JRDR, most prevalent dialysis patients are treated with hemodialysis, with only $2.7 \%$ treated with peritoneal dialysis as of the end of 2017 (3). Mortality data for the general population were obtained from the National Vital Statistics Survey in 2017 and $2018(5,6)$.

This study was conducted according to the principles of the Declaration of Helsinki, Japanese privacy protection laws, and Ethical Guidelines for Medical and Health Research Involving Human Subjects published by the Ministry of Education, Science and Culture, and the Ministry of Health, Labour and Welfare in 2015. Our analyses used existing national data without individual patient data, so consent was not required.

\section{Study Outcomes}

The cause of death among dialysis patients was defined according to the JRDR coding system and categorized according to JRDR categories (3). We defined cardiovascular mortality as death attributed to heart failure, cerebrovascular disease, myocardial infarction, or hyperkalemia/sudden death. Non-cardiovascular mortality was defined as death from all other causes, i.e. infectious disease, gastrointestinal (GI) bleeding, malignancy, cachexia/uremia/senility, liver cirrhosis, suicide/refusal of treatment, ileus, hematological disease, and pulmonary disease. Disaster or accidental deaths, others, and undetermined cases were not included in either cardiovascular or non-cardiovascular mortality categories.

Cause of death was classified using the 10th revision of the International Statistical Classification of Diseases and Related Health Problems (ICD-10) for the general population. To imitate the definition of the JRDR categories, we modified the definition of cardiovascular mortality using ICD-10 codes from a previous study (ICD-10 codes I00-I 99) (1) by adding ICD-10 codes E87 (Other disorders of fluid, electrolyte, and acid-base balance including hyperkalemia) and R96 (Other sudden death, cause unknown). Specific codes are listed in Supplemental Table S1.

\section{Statistical Analyses}

Crude mortality rates were calculated by dividing the number of deaths by the number of person-years. The number of person-years during the two-year study period was estimated by multiplying the mid-period population by the period (i.e. two years) (7-11). Age-specific rates were calculated for the following age groups: <50, 50-59, 60-69, 70$79,80-89$, and $\geq 90$ years old. Age groups were used to avoid having a small number of analyzed patients at particular ages, which could lead to a great degree of random variation (12). The MMRs were calculated using crude rates for all-cause, cardiovascular, and non-cardiovascular mortality by age group, as described in a previous study (2). Agestandardized mortality ratios (SMRs) were calculated using mortality ratios of male dialysis patients to female dialysis patients. Age-standardized mortality rates (ASRs) were then calculated using the direct method (12). The standard populations used in the present study were the population of Japanese patients on dialysis as of December 31, 2017 (3), and the entire Japanese population in 2017 for the general population (Supplemental Table S2). We calculated differences in ASRs to determine whether or not there was excess mortality in male dialysis patients compared to female dialysis patients on an absolute scale. To determine the contributions of cause-specific mortality to any differences observed in all-cause mortality, we also calculated percent differences in all-cause mortality $(8,13)$.

\section{Results}

During the 2-year study period, 62,260 deaths occurred in 643,032 person-years in the dialysis population (96.8 per 1,000 person-years). In total, non-cardiovascular disease was the cause of death in 25,957 (41.7\%), and cardiovascular disease accounted for 22,032 deaths (35.4\%). In the general population, 2,702,169 deaths were observed in 253,414,000 person-years (10.7 per 1,000 person-years). Among these, $1,622,606$ deaths $(60.0 \%)$ were from non-cardiovascular causes, and 708,003 (26.2\%) were from cardiovascular causes.

Table 1 shows the number of deaths and all-cause, cardiovascular, and non-cardiovascular mortality rates in prevalent dialysis patients stratified by sex and age category. In both 
Table 1. Number of Deaths, Person-years at Risk, and Mortality Rates Stratified by Sex and Age Category in Prevalent Dialysis Patients.

\begin{tabular}{|c|c|c|c|c|c|c|c|}
\hline & \multicolumn{3}{|c|}{ Number of deaths } & \multirow{2}{*}{$\begin{array}{l}\text { Person-years } \\
\text { at risk }\end{array}$} & \multicolumn{3}{|c|}{ Mortality rate $(95 \% \mathrm{CI})^{\mathrm{a}}$} \\
\hline & $\begin{array}{l}\text { All- } \\
\text { cause }\end{array}$ & $\mathrm{CV}$ & $\begin{array}{l}\text { Non- } \\
\text { CV }\end{array}$ & & All-cause & $\mathrm{CV}$ & Non-CV \\
\hline \multicolumn{8}{|l|}{ Males } \\
\hline All & 41,006 & 14,262 & 17,510 & 417,740 & $98.2(97.2-99.1)$ & $34.1(33.6-34.7)$ & $41.9(41.3-42.5)$ \\
\hline$<50 \mathrm{y}$ & 712 & 323 & 199 & 39,220 & $18.2(16.8-19.5)$ & $8.2(7.4-9.2)$ & $5.1(4.4-5.8)$ \\
\hline $50-59 \mathrm{y}$ & 2,114 & 937 & 631 & 61,682 & $34.3(32.8-35.8)$ & $15.2(14.2-16.2)$ & $10.2(9.4-11.1)$ \\
\hline $60-69 y$ & 7,178 & 2,669 & 2,778 & 118,874 & $60.4(59.0-61.8)$ & $22.5(21.6-23.3)$ & $23.4(22.5-24.3)$ \\
\hline $70-79 y$ & 14,094 & 4,928 & 6,059 & 124,350 & $113.3(111.5-115.2)$ & $39.6(38.5-40.8)$ & $48.7(47.5-50.0)$ \\
\hline $80-89$ y & 14,236 & 4,590 & 6,524 & 67,290 & $211.6(208.1-215.1)$ & $68.2(66.3-70.2)$ & $97.0(94.6-99.3)$ \\
\hline$\geq 90 \mathrm{y}$ & 2,663 & 815 & 1,319 & 6,324 & $421.1(405.3-437.4)$ & $128.9(120.2-138.0)$ & $208.6(197.5-220.1)$ \\
\hline \multicolumn{8}{|l|}{ Females } \\
\hline All & 21,254 & 7,770 & 8,447 & 225,292 & $94.3(93.1-95.6)$ & $34.5(33.7-35.3)$ & $37.5(36.7-38.3)$ \\
\hline$<50 \mathrm{y}$ & 266 & 96 & 94 & 16,256 & $16.4(14.5-18.5)$ & $5.9(4.8-7.2)$ & $5.8(4.7-7.1)$ \\
\hline $50-59$ y & 697 & 278 & 240 & 26,324 & $26.5(24.5-28.5)$ & $10.6(9.4-11.9)$ & $9.1(8.0-10.3)$ \\
\hline $60-69 y$ & 2,665 & 1,005 & 981 & 59,782 & $44.6(42.9-46.3)$ & $16.8(15.8-17.9)$ & $16.4(15.4-17.5)$ \\
\hline $70-79 y$ & 6,275 & 2,292 & 2,524 & 68,964 & $91.0(88.8-93.3)$ & $33.2(31.9-34.6)$ & $36.6(35.2-38.1)$ \\
\hline $80-89$ y & 8,727 & 3,209 & 3,479 & 46,942 & $185.9(182.0-189.9)$ & $68.4(66.0-70.8)$ & $74.1(71.7-76.6)$ \\
\hline$\geq 90 \mathrm{y}$ & 2,624 & 890 & 1,129 & 7,024 & $373.6(359.4-388.2)$ & $126.7(118.5-135.3)$ & $160.7(151.5-170.4)$ \\
\hline
\end{tabular}

aRates per 1,000 person-years

CI: confidence interval, $\mathrm{CV}$ : cardiovascular

male and female prevalent dialysis patients, noncardiovascular mortality rates were lower than cardiovascular mortality rates in younger age categories but increased steeply with age, resulting in non-cardiovascular mortality rates surpassing cardiovascular mortality rates in older age categories. In the general population, non-cardiovascular mortality rates surpassed cardiovascular mortality rates in all age groups (Supplemental Table S3). A graphic representation of mortality rates is provided in Fig. 1. In both sexes, all-cause, cardiovascular, and non-cardiovascular mortality rates were higher in dialysis patients than in the general population, but the difference between the two populations became smaller with increasing age, especially for noncardiovascular mortality rates. As expected, all-cause mortality in males surpassed that in females for all six age groups in the general population. Male dialysis patients also had higher all-cause mortality rates than female patients across all age categories (Table 2), although the MMR was smaller than that observed in the general population (Fig. 1d). In both the dialysis and general populations, MMRs for cardiovascular disease approached 1 in older age categories (Fig. 1e). In contrast, MMRs for non-cardiovascular disease were large in older age categories (Fig. 1f).

All-cause mortality rates in male dialysis patients were higher than those in females, with a relative risk of 1.21 (95\% confidence interval [CI], 1.20-1.23) after adjusting for age (Table 3). SMRs for cardiovascular and noncardiovascular diseases were 1.15 (95\% CI: 1.13-1.17) and 1.32 (95\% CI: 1.30-1.34) times higher, respectively, in male dialysis patients compared to female dialysis patients. The ASR difference for cardiovascular diseases (3.2 per 1,000 person-years) was about one third of that for non- cardiovascular diseases (9.7 per 1,000 person-years). Death from cardiovascular and non-cardiovascular diseases contributed to $21.4 \%$ and $63.8 \%$ of the difference in all-cause mortality, respectively.

SMRs for cause-specific mortality were significantly higher in males than in females for disaster or accidental deaths, pulmonary disease, malignancy, suicide/refusal of treatment, myocardial infarction, infectious disease, hyperkalemia/sudden death, undetermined, liver cirrhosis, and heart failure, in descending order. In contrast, SMRs for cachexia/ uremia/senility and ileus were significantly lower in males than in females. There were no significant differences between sexes for cerebrovascular disease, GI bleeding, hematological disease, and others. On an absolute scale, ASR differences were large for infectious disease (5.8 per 1,000 person-years), malignancy (3.4 per 1,000 person-years), undetermined (2.4 per 1,000 person-years), and heart failure (1.8 per 1,000 person-years), in descending order. Death from infectious disease, malignancy, undetermined, and heart failure contributed to $38.4 \%, 22.7 \%, 15.9 \%$, and $12.1 \%$, respectively, of the difference in all-cause mortality.

Fig. 2 shows the age-specific mortality rates in dialysis patients stratified by sex. While many cause-specific mortality rates were higher in male dialysis patients than in female dialysis patients, mortality rates of ileus and cachexia/uremia/senility were lower in males than in females. Notably, stroke mortality rates were higher in young male dialysis patients than in their female counterparts, whereas the rates were lower in older male dialysis patients than in their female counterparts. This finding was not observed in the general population, with males having higher stroke mortality rates than females across all age categories (Fig. 3). Mortal- 

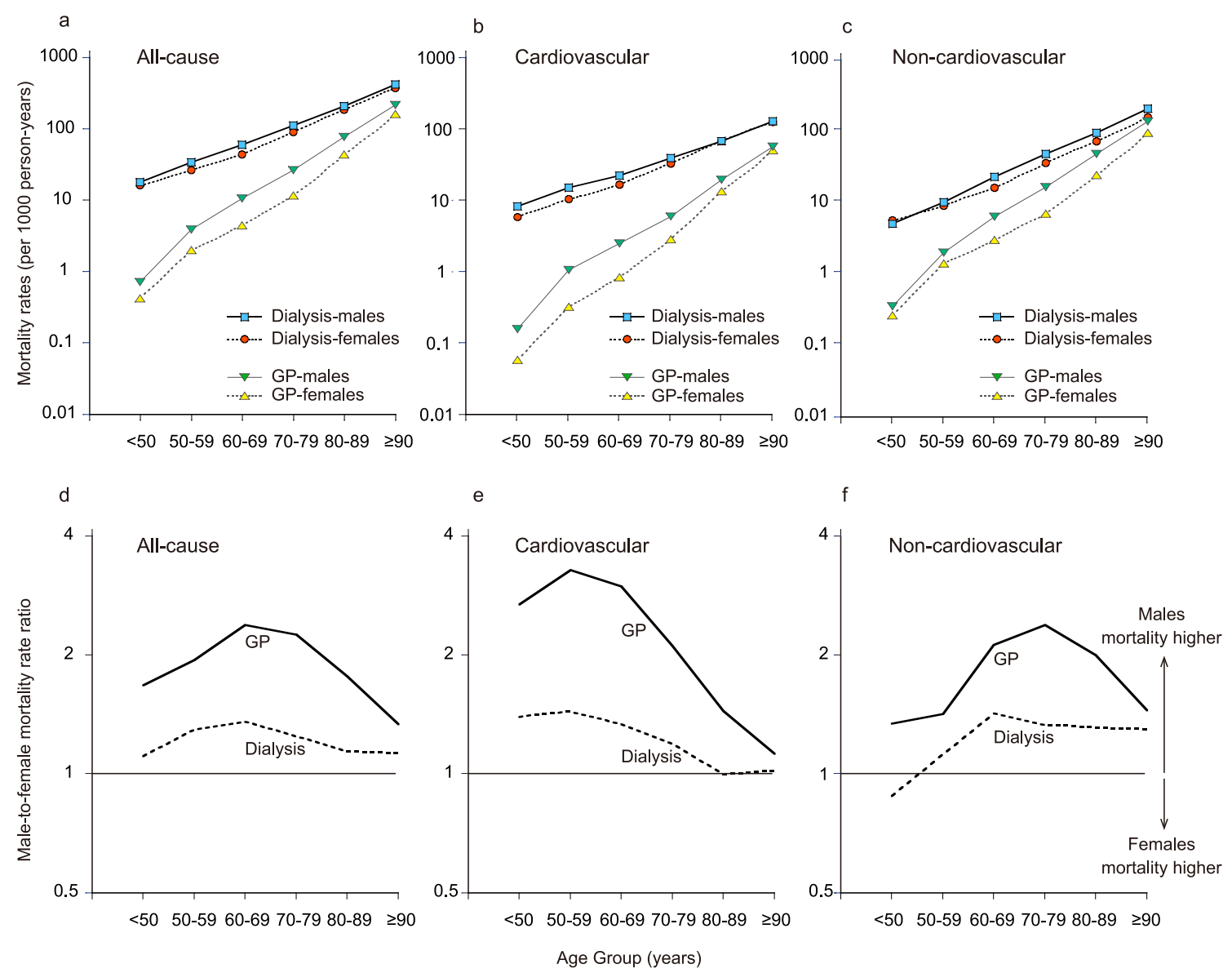

Figure 1. Sex-specific mortality rates and male-to-female mortality rate ratios (MMRs). (a) Allcause (b) cardiovascular, and (c) non-cardiovascular sex-specific mortality rates (logarithmic scale) and (d) all cause, (e) cardiovascular, and (f) non-cardiovascular MMRs in the dialysis population and general population by age category. GP: general population, MMR: male-to-female mortality rate ratio

Table 2. Male-to-female Mortality Rate Ratios for All-cause, CV, and Non-CV Mortality among Prevalent Dialysis Patients by Age Group.

\begin{tabular}{lccc}
\hline & \multicolumn{3}{c}{ Male-to-female mortality rate ratios (95\% CI) } \\
\cline { 2 - 4 } & All-cause & CV & Non-CV \\
\hline$<50$ y & $1.11(1.09$ to 1.13$)$ & $1.39(1.37$ to 1.42$)$ & $0.88(0.86$ to 0.89$)$ \\
$50-59$ y & $1.29(1.28$ to 1.31$)$ & $1.44(1.42$ to 1.46$)$ & $1.12(1.11$ to 1.14$)$ \\
$60-69$ y & $1.36(1.34$ to 1.37$)$ & $1.34(1.32$ to 1.35$)$ & $1.42(1.41$ to 1.44$)$ \\
$70-79$ y & $1.25(1.23$ to 1.26$)$ & $1.19(1.18$ to 1.20$)$ & $1.33(1.32$ to 1.34$)$ \\
$80-89$ y & $1.14(1.12$ to 1.15$)$ & $1.00(0.99$ to 1.01$)$ & $1.31(1.29$ to 1.32$)$ \\
$\geq 90$ y & $1.13(1.09$ to 1.17$)$ & $1.02(0.98$ to 1.05$)$ & $1.30(1.25$ to 1.12$)$ \\
\hline
\end{tabular}

Mortality rate ratios are unadjusted.

CI: confidence interval, CV: cardiovascular

ity rates due to ileus were higher in males than in females in the general population (Supplemental Table S4).

\section{Discussion}

The present study demonstrated that all-cause mortality in males surpassed that of females in both the dialysis and general populations, although the MMR in the dialysis population was smaller than that observed in the general population. This female survival advantage was mainly attributed to cardiovascular mortality in younger age categories and non-cardiovascular mortality in older age categories in both the dialysis and general populations. Infectious disease and malignancy among non-cardiovascular diseases and heart failure among cardiovascular diseases were the main contributors to the sex difference in all-cause mortality, sug- 
Table 3. Sex-specific Causes of Death and Standardized Mortality Ratios among Prevalent Dialysis Patients.

\begin{tabular}{|c|c|c|c|c|c|c|c|}
\hline \multirow[b]{2}{*}{ Cause of death } & \multicolumn{2}{|c|}{ Female patients } & \multicolumn{2}{|c|}{ Male patients } & \multirow{2}{*}{$\begin{array}{l}\mathrm{SMR}^{\mathrm{b}} \text { of male } \\
\text { patients } \\
\text { compared with } \\
\text { female patients } \\
(95 \% \mathrm{CI})\end{array}$} & \multicolumn{2}{|c|}{ Male-to-female difference ${ }^{c}$} \\
\hline & $\begin{array}{l}\text { Total } \\
\text { No. of } \\
\text { deaths }\end{array}$ & $\begin{array}{c}\text { Crude } \\
\text { mortality } \\
\text { rates }^{\mathrm{a}}\end{array}$ & $\begin{array}{l}\text { Total } \\
\text { No. of } \\
\text { deaths }\end{array}$ & $\begin{array}{l}\text { Crude } \\
\text { mortality } \\
\text { rates }^{\text {a }}\end{array}$ & & $\begin{array}{c}\text { Age-adjusted } \\
\text { mortality rate } \\
\text { difference a }^{\text {a }}\end{array}$ & $\begin{array}{c}\% \text { difference } \\
\text { in all-cause } \\
\text { mortality }\end{array}$ \\
\hline Cardiovascular diseases & 7,770 & 34.5 & 14,262 & 37.5 & $1.15(1.13-1.17)$ & 3.2 & 21.4 \\
\hline Heart failure & 5,365 & 23.8 & 9,420 & 22.5 & $1.12(1.10-1.15)$ & 1.8 & 12.1 \\
\hline Cerebrovascular disease & 1,393 & 6.2 & 2,319 & 5.6 & $1.00(0.96-1.04)$ & -0.1 & -0.8 \\
\hline Myocardial infarction & 657 & 2.9 & 1,673 & 4.0 & $1.53(1.46-1.61)$ & 1.1 & 7.3 \\
\hline Hyperkalemia / Sudden death & 355 & 1.6 & 850 & 2.0 & $1.36(1.27-1.45)$ & 0.4 & 2.7 \\
\hline Non-cardiovascular diseases & 8,447 & 37.5 & 17,510 & 41.9 & $1.32(1.30-1.34)$ & 9.7 & 63.8 \\
\hline Infectious disease & 4,195 & 18.5 & 9,037 & 21.6 & $1.37(1.34-1.40)$ & 5.8 & 38.4 \\
\hline GI bleeding & 315 & 1.4 & 507 & 1.2 & $0.99(0.90-1.08)$ & 0.0 & -0.3 \\
\hline Malignancy & 1,447 & 6.4 & 3,964 & 9.5 & $1.63(1.58-1.68)$ & 3.4 & 22.7 \\
\hline Cachexia / Uremia / senility & 1,542 & 6.8 & 1,858 & 4.4 & $0.88(0.84-0.92)$ & -0.8 & -5.0 \\
\hline Liver cirrhosis & 116 & 0.5 & 275 & 0.7 & $1.31(1.16-1.48)$ & 0.1 & 0.7 \\
\hline Suicide / Refusal of treatment & 105 & 0.5 & 268 & 0.6 & $1.59(1.41-1.79)$ & 0.2 & 1.3 \\
\hline Ileus & 263 & 1.2 & 346 & 0.8 & $0.79(0.71-0.88)$ & -0.1 & -0.6 \\
\hline Hematological disease & 89 & 0.4 & 157 & 0.4 & $1.10(0.93-1.28)$ & 0.0 & 0.2 \\
\hline Pulmonary disease & 411 & 1.8 & 1,098 & 2.6 & $1.66(1.57-1.77)$ & 1.1 & 7.2 \\
\hline Disaster or accidental deaths & 63 & 0.3 & 205 & 0.5 & $1.90(1.65-2.18)$ & 0.2 & 1.2 \\
\hline Others & 2,605 & 11.6 & 3,949 & 9.5 & $0.97(0.94-1.00)$ & -0.4 & -2.4 \\
\hline Undetermined & 2,369 & 10.5 & 5,080 & 12.2 & $1.32(1.28-1.35)$ & 2.4 & 15.9 \\
\hline All-cause mortality & 21,254 & 94.3 & 41,006 & 98.2 & $1.21(1.20-1.23)$ & 15.2 & 100.0 \\
\hline
\end{tabular}

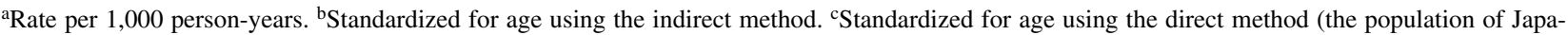
nese patients on dialysis as of December 31, 2017 was used as the standard population).

CI: confidence interval, GI: gastrointestinal, SMR: standardized mortality ratio. All percentages may not total $100 \%$ due to rounding.

gesting that the female survival advantage in the dialysis population is attributable to these diseases. Sex-specific mortality in the dialysis population also differed by cause of death. Our findings may contribute to the designing of strategies to reduce the high mortality rate in dialysis patients.

While our findings do not explain why there is a female survival advantage among Japanese dialysis patients, there are a number of possibilities. First, the lower mortality rate for infectious disease in Japanese female dialysis patients relative to their male counterparts may be a factor. We found that infectious diseases contributed to $38.4 \%$ of the male-tofemale difference in all-cause mortality, suggesting that infectious disease is the largest contributor to the female survival advantage among Japanese dialysis patients. However, a cohort study of patients starting renal replacement therapy using data from the ERA-EDTA registry showed that female sex was associated with an increased risk of infectionrelated mortality (14), which is more consistent with loss of the female survival advantage. Since infection is the leading cause of non-cardiovascular mortality in dialysis patients in Europe, the United States, and Japan (15), infection is likely to have a major impact on the survival advantage.

Second, the female survival advantage may result from the lower prevalence of diabetes in Japanese female dialysis patients relative to their male counterparts. According to two previous studies, the interaction effect of diabetes may explain the attenuated survival advantage in female dialysis patients $(16,17)$. A prospective cohort study using data from the Australia and New Zealand Dialysis and Transplant (ANZDATA) Registry reported that female sex was significantly associated with a higher risk for death in type 2 diabetes patients, but not in type 1 diabetes and in non-diabetic patients (16). Another prospective cohort study of incident dialysis patients using data from the Netherlands Cooperative Study on the Adequacy of Dialysis (NECOSAD) similarly showed that both all-cause and cardiovascular mortality rates were higher in diabetic women than in diabetic men (17). Unlike other countries, Japanese female dialysis patients are less likely to have diabetes than their male counterparts. For instance, the DOPPS study reported that the proportions of male and female prevalent hemodialysis patients for whom diabetes was the cause of ESKD were $19.7 \%$ and $20.9 \%$, respectively, for Europe and Australia/ New Zealand; $37.1 \%$ and $43.6 \%$, respectively, for North America; and $29.2 \%$ and $21.3 \%$, respectively, for Japan (2). The JRDR reported that the proportions of male and female prevalent dialysis patients for whom diabetic nephropathy 

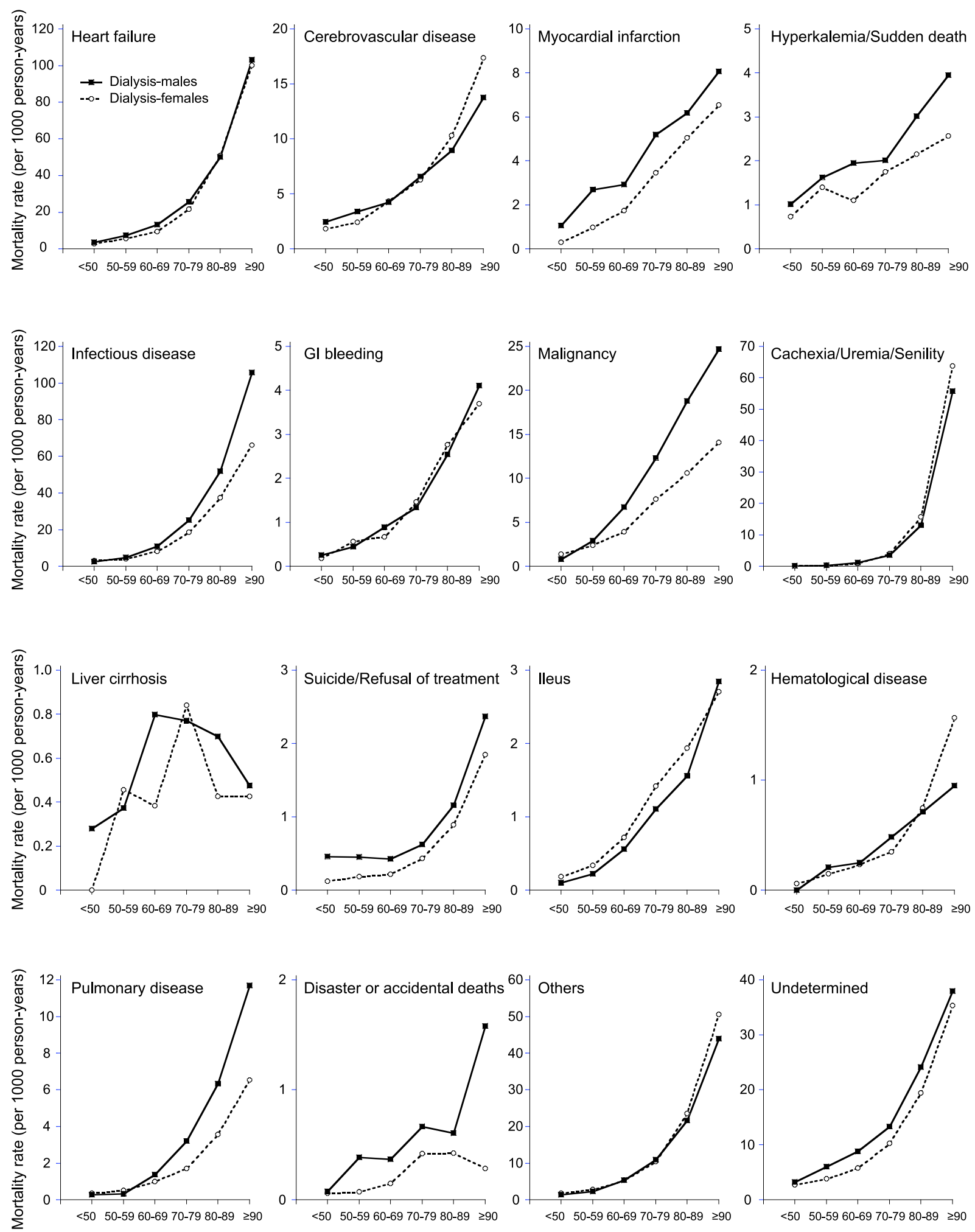

Age Group (years)

Figure 2. Cause-specific mortality rates stratified by age in male (solid line and filled squares) and female (dashed line and open circles) prevalent dialysis patients. GI: gastrointestinal

was the cause of ESKD were $42.7 \%$ and $39.0 \%$, respectively, as of the end of 2017 (3). Taken together, Japanese female dialysis patients are less likely to have diabetes, and this may contribute to the survival advantage of female dialysis patients over their male counterparts in Japan.

Third, the better survival of the Japanese dialysis popula- tion (18-20) may dilute the effect of dialysis as a "great equalizer" (2). Hecking et al. (2) speculated that hemodialysis could be viewed as a "great equalizer" because of the hemodialysis mortality rate being 10 to 20 times higher than in the general population. Thus, differences between males and females that have an important impact in the general 

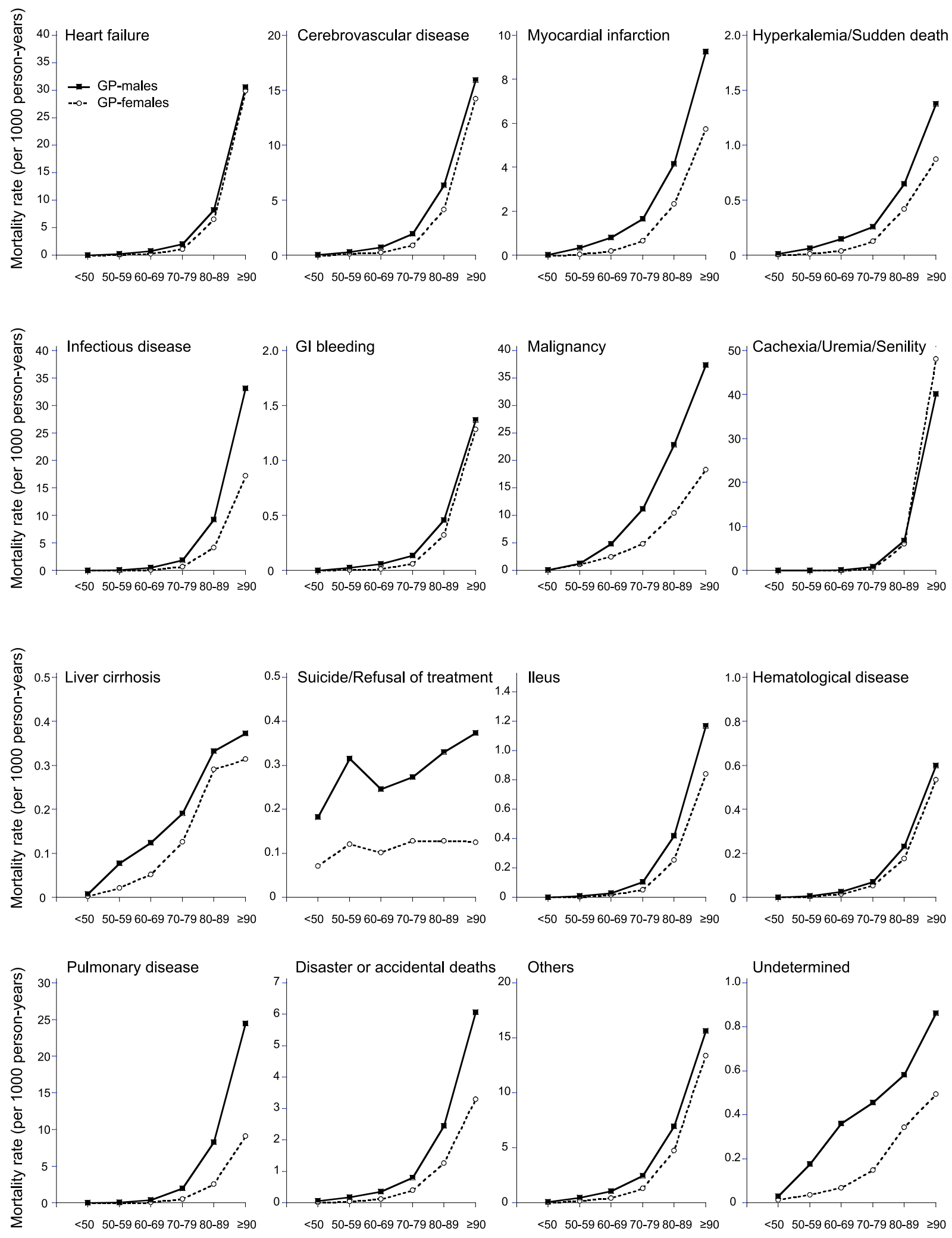

Age Group (years)

Figure 3. Cause-specific mortality rates stratified by age in males (solid line and filled squares) and females (dashed line and open circles) of the general population. GI: gastrointestinal

population might lose importance in dialysis patients (2). In Japan, the crude all-cause mortality of dialysis patients is around 94 per 1,000 person-years $(3,4,9)$, and the SMR for all-cause mortality was 4.6 (95\% CI, 4.6-4.7) for dialysis patients compared to the general population (9). Given a di- alysis mortality rate five times higher than in the general population, the effect of dialysis as a "great equalizer" may be weaker in Japan than in other countries, thereby preserving the survival advantage in Japanese female dialysis patients. 
Finally, some common mechanisms might exist between Japanese dialysis patients and general populations. This is supported by our results showing that the female survival advantage was mainly attributed to cardiovascular mortality in younger age categories and non-cardiovascular mortality in older age categories in both populations. Thus, certain preventable factors specific to Japan might be shared between the dialysis and general populations.

Sex-specific mortality in dialysis patients was found to vary by cause of death. Among the several interesting findings related to cause-specific mortality, we focus here on the sex-specific mortality due to cerebrovascular disease, which showed differences with regard to the age and influence of sex. Specifically, mortality rates were higher in young male dialysis patients than their female counterparts, whereas the rates were lower in older male dialysis patients than their female counterparts. This was not observed in the general population, suggesting that the underlying mechanisms are related to dialysis. One possible reason for this finding is that our study calculated the overall cerebrovascular disease mortality, rather than handling intracranial hemorrhage and cerebral infarction separately. Using JRDR data, we previously reported that mortality rates for intracranial hemorrhage were markedly higher in dialysis patients compared with the general population, even among younger age groups, whereas mortality rates for cerebral infarction steeply increased in older age categories (10). This suggests that mortality rates due to cerebrovascular disease may be attributed mainly to intracranial hemorrhage in younger age categories and to cerebral infarction in older age categories. Furthermore, the results of the Q-cohort study, a prospective cohort study of Japanese prevalent dialysis patients, showed sex differences for stroke risk factors. That study found that lower phosphate levels increased the risk of brain infarction in female patients (hazard ratio [HR] 2.23; 95\% CI, 1.01 to 5.05 ) but not in male patients (HR $0.92 ; 95 \% \mathrm{CI}, 0.50$ to 1.72; $P$ for interaction 0.016 ). In contrast, higher phosphate levels increased the risk of brain hemorrhage in male patients (HR 2.80; 95\% CI, 1.35 to 5.59) but not in female patients (HR 0.97; 95\% CI, 0.27 to 3.29), although the interaction was not significant ( $P$ for interaction 0.150$)(21)$. The different age and sex effects on stroke mortality observed in our study might be explained by the different effects of serum phosphate levels on stroke incidence by sex.

With regard to the association between sex and stroke mortality, previous studies have reported inconsistent findings. Male sex was a significant risk factor for death after a stroke (adjusted subhazard ratio $=1.41$ [95\% CI, 1.21 to 1.64]) in a cohort study of dually-eligible (Medicare and Medicaid) dialysis patients (22). In contrast, female sex was a significant risk factor for stroke death (adjusted hazard ratio=1.04 [95\% CI, 1.01 to 1.08]) in a cohort of incident ESKD patients using data from the ANZDATA registry (23). These inconsistent findings may be related to the different age and sex effects on stroke mortality.

This study has several strengths. First, we used data from a national Vital Statistics survey and a nationwide survey of Japanese dialysis facilities. Both surveys are an almost complete national census. Second, we comprehensively evaluated the sex- and cause-specific mortality among Japanese dialysis and general populations.

However, several limitations associated with the present study also warrant mention. First, the different classification of causes of death used in the general and dialysis populations may have introduced misclassification bias. Second, we adjusted only for age and sex. In the DOPPS study, however, despite substantial cross-sectional differences between males and females on hemodialysis, these adjustments altered the crude MMR only slightly (2). Finally, as this study was conducted in Japan, the results might not be generalizable to other populations. However, since a small excess mortality risk for males over females is also observed in some European countries (2), similar results may be observed in other populations.

\section{Conclusions}

The present nationwide study of Japanese dialysis patients confirmed the female survival advantage in Japanese dialysis patients. Our findings also add evidence that the female survival advantage is mainly attributed to cardiovascular mortality in younger age categories and non-cardiovascular mortality in older age categories. The sex-specific mortality in dialysis patients was also found to differ by cause of death. Our results expand on the existing knowledge and understanding of sex differences in mortality seen in dialysis patients, which is a necessary step in designing strategies to reduce the high mortality rate in this patient population.

The authors state that they have no Conflict of Interest (COI).

\section{Acknowledgement}

We thank the Committee of the Renal Data Registry of the Japanese Society for Dialysis Therapy (JSDT) for permission to use their registry data. The results in the current study were derived from split data from the WADDA system of the JSDT by the authors. However, the interpretation and reporting of these data are the responsibility of the authors and in no way should be seen as the official policy or interpretation of the JSDT.

\section{Funding}

This work was supported by a research grant from JSPS KAKENHI (Grants-in-Aid for Scientific Research, Grant Number JP18K08202) to MW. No funding agency had any role in the study design, collection, analysis, or interpretation of data, writing the report, or the decision to submit the report for publication.

\section{References}

1. Carrero JJ, de Jager DJ, Verduijn $M$, et al. Cardiovascular and noncardiovascular mortality among men and women starting dialysis. Clin J Am Soc Nephrol 6: 1722-1730, 2011. 
2. Hecking M, Bieber BA, Ethier J, et al. Sex-specific differences in hemodialysis prevalence and practices and the male-to-female mortality rate: the Dialysis Outcomes and Practice Patterns Study (DOPPS). PLoS Med 11: e1001750, 2014.

3. Nitta K, Masakane I, Hanafusa N, et al. Annual dialysis data report 2017, JSDT Renal Data Registry. Ren Replace Ther 5: 53, 2019. https://doi.org/10.1186/s41100-019-0248-1

4. Nitta K, Goto S, Masakane I, et al. Annual dialysis data report for 2018, JSDT Renal Data Registry: survey methods, facility data, incidence, prevalence, and mortality. Ren Replace Ther 6: 41, 2020. https://doi.org/10.1186/s41100-020-00286-9

5. Bureau of Census, Ministry of Internal Affairs and Communications. Vital statistics of Japan [Internet]. 2017 [cited 2021 Jul 7]. Available from: https://www.e-stat.go.jp/stat-search/files?page=1\&1 ayout $=$ datalist $\&$ toukei $=00450011 \&$ tstat $=000001028897 \&$ cycle $=7 \&$ year $=20170 \&$ month $=0 \&$ tclass $1=000001053058 \&$ tclass $2=00000105$ $3061 \&$ tclass $3=000001053065 \&$ result_back $=1 \&$ tclass 4 val $=0$

6. Bureau of Census, Ministry of Internal Affairs and Communications. Vital statistics of Japan [Internet]. 2018 [cited 2021 Jul 7]. Available from: https://www.e-stat.go.jp/stat-search/files?page=1\&1 ayout $=$ datalist $\&$ toukei $=00450011 \&$ tstat $=000001028897 \&$ cycle $=7 \&$ year $=20180 \&$ month $=0 \&$ tclass $1=000001053058 \&$ tclass $2=00000105$ $3061 \&$ tclass $3=000001053065 \&$ result_back $=1 \&$ tclass 4 val $=0$

7. Esteve J, Benhamou E, Raymond L. Techniques for the analysis of cancer risk. In: Statistical Methods In Cancer Research: Volume IV: Descriptive Epidemiology. Esteve J, Benhamou E, Raymond L, Eds. International Agency for Research on Cancer, Lyon, 1994: 49-105.

8. Wakasugi M, Kawamura K, Yamamoto S, et al. High mortality rate of infectious diseases in dialysis patients: a comparison with the general population in Japan. Ther Apher Dial 16: 226-231, 2012.

9. Wakasugi M, Kazama JJ, Yamamoto S, et al. Cause-specific excess mortality among dialysis patients: comparison with the general population in Japan. Ther Apher Dial 17: 298-304, 2013.

10. Wakasugi M, Matsuo K, Kazama JJ, et al. Higher Mortality Due to Intracerebral Hemorrhage in Dialysis Patients: A Comparison with the General Population in Japan. Ther Apher Dial 19: 45-49, 2014.

11. Wakasugi M, Kazama JJ, Narita I. Mortality trends among Japanese dialysis patients, 1988-2013: a joinpoint regression analysis. Nephrol Dial Transplant 31: 1501-1507, 2016.

12. Curtin LR, Klein RJ. Direct standardization (age-adjusted death rates). Healthy People 2000 Stat Notes 1-10, 1995.

13. Richardus JH, Kunst AE. Black-white differences in infectious dis- ease mortality in the United States. Am J Public Health 91: 12511253, 2001.

14. Vogelzang JL, van Stralen KJ, Noordzij M, et al. Mortality from infections and malignancies in patients treated with renal replacement therapy: data from the ERA-EDTA registry. Nephrol Dial Transplant 30: 1028-1037, 2015.

15. de Jager DJ, Vervloet MG, Dekker FW. Noncardiovascular mortality in CKD: an epidemiological perspective. Nat Rev Nephrol 10: 208-214, 2014.

16. Villar E, Chang SH, McDonald SP. Incidences, treatments, outcomes, and sex effect on survival in patients with end-stage renal disease by diabetes status in Australia and New Zealand (1991 2005). Diabetes Care 30: 3070-3076, 2007.

17. Carrero JJ, de Mutsert R, Axelsson J, et al. Sex differences in the impact of diabetes on mortality in chronic dialysis patients. Nephrol Dial Transplant 26: 270-276, 2011.

18. Goodkin DA, Bragg-Gresham JL, Koenig KG, et al. Association of comorbid conditions and mortality in hemodialysis patients in Europe, Japan, and the United States: the Dialysis Outcomes and Practice Patterns Study (DOPPS). J Am Soc Nephrol 14: 32703277, 2003.

19. Robinson BM, Akizawa T, Jager KJ, et al. Factors affecting outcomes in patients reaching end-stage kidney disease worldwide: differences in access to renal replacement therapy, modality use, and haemodialysis practices. Lancet 388: 294-306, 2016.

20. Yoshino M, Kuhlmann MK, Kotanko P, et al. International differences in dialysis mortality reflect background general population atherosclerotic cardiovascular mortality. J Am Soc Nephrol 17: 3510-3519, 2006.

21. Yamada S, Tsuruya K, Taniguchi M, et al. Association Between Serum Phosphate Levels and Stroke Risk in Patients Undergoing Hemodialysis: The Q-Cohort Study. Stroke 47: 2189-2196, 2016.

22. Wetmore JB, Phadnis MA, Ellerbeck EF, et al. Relationship between stroke and mortality in dialysis patients. Clin J Am Soc Nephrol 10: 80-89, 2015.

23. De La Mata NL, Alfaro-Ramirez M, Kelly PJ, et al. Absolute risk and risk factors for stroke mortality in patients with end-stage kidney disease (ESKD): population-based cohort study using data linkage. BMJ Open 9: e026263, 2019.

The Internal Medicine is an Open Access journal distributed under the Creative Commons Attribution-NonCommercial-NoDerivatives 4.0 International License. To view the details of this license, please visit (https://creativecommons.org/licenses/ by-nc-nd/4.0/).

(C) The Japanese Society of Internal Medicine Intern Med Advance Publication 